\title{
¿Cómo definir sustantivos que designan sentimientos/ emociones? Análisis de paráfrasis y apreciación de propuestas para su perfeccionamiento
}

\section{How to define emotion nouns? Analysis of paraphrases and assessment of proposals for its further development}

Virginia Sita Farias

UFRJ

virginiafarias@hotmail.com

Resumen: Este artículo trata de las dificultades que se plantean en la definición de sustantivos que designan sentimientos/emociones. Primero se analizan paráfrasis tomadas de diccionarios de español, portugués, italiano y francés, a fin de trazar un panorama de los mecanismos definitorios a los que se recurre para definir este tipo de unidades léxicas. A continuación se evalúan los aportes de dos modelos semánticos -el metalenguaje semántico natural y la semántica de marcos- a la generación de definiciones de sustantivos que designan sentimientos/ emociones. La apreciación crítica de las teorías semánticas es el primer paso hacia la elaboración de una propuesta para el perfeccionamiento de las definiciones de sustantivos que designan sentimientos/emociones en los diccionarios de lengua.

Palabras-clave: (meta)lexicografía; definición lexicográfica; sentimientos; emociones. 
Abstract: This paper deals with the problems inherent to the definition of emotion nouns. Firstly, we analyze definitions taken from Spanish, Portuguese, Italian and French dictionaries to draw a panorama of explanatory mechanisms that dictionary-makers use to explain emotion concepts. Next, we evaluate the contributions of two theoretical models - the Natural Semantic Metalanguage and the Frame Semantics - to the creation of definitions for emotion nouns. The critical assessment of semantic theories is the first step in the preparation of a proposal for the improvement of definitions of emotion nouns in language dictionaries.

Keywords: (meta)lexicography; lexicographic definition; feelings; emotions.

Recebido em 19 de junho de 2015. Aprovado em 31 de agosto de 2015.

\section{Introducción}

Seco (2003, p. 30-31) advierte que "la definición es, a la vez que la médula del artículo lexicográfico, la tarea más ardua que le toca al lexicógrafo". Porto Dapena (2002, p. 266-267) corrobora esas palabras y añade que la generación de paráfrasis explicativas sigue siendo "el principal escollo dentro de la redacción lexicográfica y, al mismo tiempo, el punto sobre el que se han venido centrando en buena medida las críticas dirigidas al diccionario monolingüe tradicional".

La importancia de la definición y las dificultades inherentes a su formulación justifican la abundante bibliografía que examina el problema desde distintas perspectivas; a modo de ilustración, mencionamos a Casares (1992), Rey-Debove (1966; 1971), Pottier (1977), Haensch et al. (1982), Wiegand (1989), Lara (1996), Geeraerts (2003), Hanks (2003) y Rundell (2008). A pesar de ello, Bugueño Miranda (2009) llama la atención sobre la ausencia -casi paradójica- de una "teoría integral de la definición lexicográfica", hecho que contribuye a explicar porque todavía es posible encontrar definiciones deficitarias aun en los mejores diccionarios de las más importantes tradiciones lexicográficas ${ }^{1}$.

${ }^{1}$ Ejemplos de definiciones deficitarias: multiplicidad "Multitud, abundancia excesiva de algunos hechos, especies o individuos" (DRAEe, 2001, s.v., ac. 2); chlorofluorocarbon 
Bugueño Miranda (2009) postula que una "teoría integral de la definición" debería anclarse en tres parámetros: (a) una taxonomía de paráfrasis, (b) un pattern sintáctico y (c) una teoría semántica.

La taxonomía de paráfrasis permite ofrecer una imagen de conjunto de la variada gama de opciones de reescritura que comporta el lado derecho de la ecuación lexicográfica. La generación de patterns sintácticos, a su vez, posibilita obtener modelos de redacción distintos para cada tipo específico de signo-lema. Por fin, la teoría semántica constituye un instrumento heurístico que actúa tanto en la delimitación del contenido proposicional de la paráfrasis como en la determinación de su potencial explicativo ${ }^{2}$.

Este trabajo tiene el objetivo de contribuir a la descripción de las relaciones entre teoría(s) semántica(s) y definición lexicográfica. Con este propósito, se analizan, en primer lugar, paráfrasis tomadas de diccionarios de español, portugués, italiano y francés, a fin de trazar un panorama -aunque parcial- de los mecanismos definitorios a los que se recurre para definir sustantivos que designan sentimientos/ emociones. A continuación se evalúan los aportes de dos modelos teóricos -el metalenguaje semántico natural y la semántica de marcosa la generación de definiciones de este tipo de unidad léxica.

Circunscribiéndose al ámbito del proyecto de investigación que aspira a proponer las bases de una "teoría integral de la definición"”, este artículo -de carácter exploratorio y descriptivopresenta los resultados obtenidos en lo concerniente a la selección de una teoría semántica que pueda subsidiar la redacción de paráfrasis de sustantivos que designan sentimientos/emociones, partiendo del análisis de algunos ejemplos. No se tiene, pues, la pretensión -al menos en este primer momento- de presentar una propuesta acabada y cerrada para la formulación de las definiciones.

\footnotetext{
"a CFC; a compound containing carbon fluorine and chlorine that is harmful to the ozone layer" (OALD, 2005, s.v.).

${ }^{2}$ Sobre la taxonomía de paráfrasis y el pattern sintáctico, cf. Bugueño Miranda (2009) y Bugueño Miranda y Farias (2011a); sobre la relación entre teoría semántica y definición lexicográfica, cf. Farias (2012) y Bugueño Miranda y Farias (2011b, 2013a, 2013b). ${ }^{3}$ Para una descripción de los objetivos, la metodología y los resultados obtenidos hasta el presente momento en el marco del referido proyecto, cf. Farias $(2010,2013)$ y Bugueño Miranda y Farias (2011a).
} 


\section{Fundamentos de una "teoría de la definición lexicográfi- ca": La distinción entre lengua y mundo en los ámbitos semántico y lexicográfico}

Ya el famoso triángulo name-sense-thing, de Ullmann (1964), cuya forma exterior se remonta a Ogden yRichards (1956), le restituye al signo lingüístico dual -constante de significante y significadoalgo que es inherente a su esencia: el referente extralingüístico. En el ámbito de la semántica estructural, Baldinger (1977) adopta como base metodológica el triángulo de Ullmann (1964), reinterpretando sus tres vértices en términos de significante (imagen acústica)-concepto (objeto mental)-realidad. Sin embargo, Heger le reprocha a Baldinger el hecho de haber desnaturalizado el sentido primigenio del triángulo, al trasladarlo del nivel de la primera metalengua al nivel de la segunda metalengua. Baldinger (1977, p. 162) toma en cuenta la crítica, reconociendo que "en el triángulo modificado se ha perdido la consustancialidad cuantitativa entre significado y significante", destruyéndose "la unidad saussuriana del signo lingüístico".

Heger concibe su modelo de trapecio, que al trasladar "correctamente" el triángulo de Ullmann (1964) al nivel de la segunda metalengua permite, por una parte, restituir la unidad del signo lingüístico y, por otra, analizar el contenido, dando lugar a los sememas y semas (BALDINGER, 1977, p. 162-163):

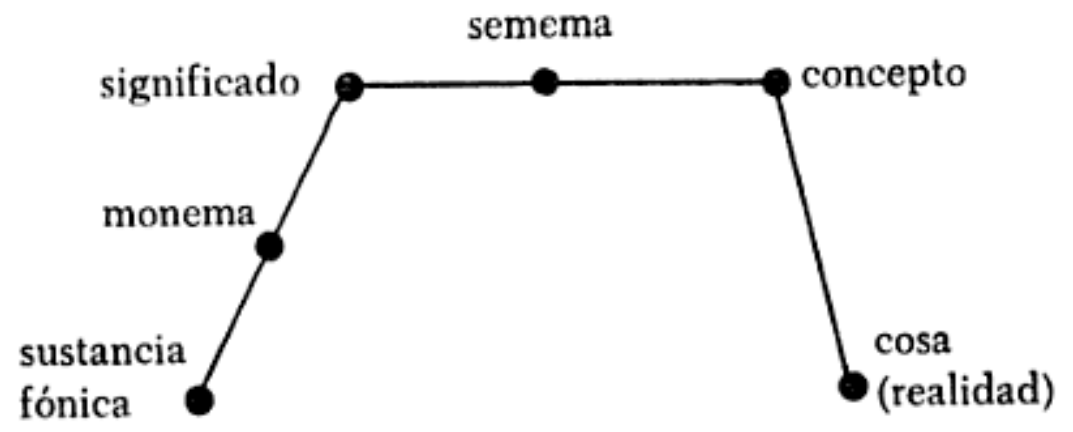

Figura 1 - Trapecio de Heger

Fuente: Baldinger (1977, p. 162) 
Según la concepción de Heger, el significado está sujeto a la estructura de una lengua particular, mientras que el concepto es independiente ${ }^{4}$. La significación $-\mathrm{o}$ semema, como prefiere- asocia significado y concepto. La realidad es, a su vez, un continuum: "[P]or lo general, la realidad no conoce límites, sino gradaciones sin límites. En estos casos -y es el caso normal- los límites no existen en la realidad, sino en el lenguaje" (BALDINGER, 1977, p. 55). ${ }^{5}$ La unión del plano estrictamente lingüístico con la realidad está mediada por la representación conceptual; el concepto está, pues, en relación directa con la realidad y constituye una suerte de "esquema de representación" de la realidad (BALDINGER, 1977, p. 50-55).

En el ámbito lexicográfico, Rey (1977,p. 100) se remonta una vez más, en el marco del estructuralismo, al triángulo de Ogden y Richards (1956), y señala que una definición, a la vez que describe un significado, apunta -aunque indirectamente- hacia un referente extralingüístico. La definición, pues, debería presentar -aunque no siempre lo hace, como veremos a continuación- una contraparte intensional y otra extensional. El definiens, que corresponde a un conjunto de condiciones necesarias y

${ }^{4}$ El concepto "ingerir [algo]" es, por así decirlo, “universal”. El español presenta dos realizaciones léxicas: beber para el significado "ingerir [algo líquido]" y comer para el significado "ingerir [algo sólido]", mientras que hay lenguas que no hacen esa distinción. El alemán, a su vez, presenta tres realizaciones léxicas: trinken para el significado "ingerir [algo líquido]", essen para el significado "ingerir [algo sólido] (en se tratando de personas)" y fressen para el significado "ingerir [algo sólido] (en se tratando de animales)". Para citar un ejemplo más: al concepto "deseo vehemente de tener la posesión de [algo/alguien] para sí solo" corresponden, en español, las designaciones envidia (respecto a algo/alguien que "esté en posesión" de otra persona) y celo(s) (respecto a algo de que se tiene la posesión/a alguien de quien se juzga tener la posesión); el árabe, por el contrario -y hasta donde yo sepa-, no coincide con el español en esta distinción.

${ }^{5}$ Un ejemplo ya clásico en la literatura respecto de este tema es el espectro de los colores. Las lenguas no siempre coinciden en la segmentación que hacen al interior del espectro (el beréber, por ejemplo, presenta una única designación para los colores azul y verde; el ruso, a su vez, establece una delimitación entre el azul celeste y el azul); ello, sin embargo, no quiere decir que un individuo perteneciente a un pueblo beréber del Sahara no sea capaz de percibir la gradación de los colores que va desde el verde oscuro hasta el azul claro, o, asimismo, que un hablante nativo del español no pueda distinguir ópticamente entre el azul claro y el azul oscuro. 
suficientes para que una entidad $x$ pertenezca a la categoría $Y$ exprime la intensión del definiendum; la equivalencia entre definiendum y definiens -la "ecuación sémica" [équation sémique] (REY-DEBOVE, 1966)- se establece si, y solamente si, la proposición que exprime el definiens tenga exactamente la misma referencia (extensión) que el definiendum.

Con la emergencia de la lingüística generativa en los años $1960 \mathrm{y}$, sobre todo, de la llamada "lingüística cognitiva", en la década siguiente, el significado -al igual que en la tradición histórico-filológica- vuelve a ser entendido como una suerte de representaciones de "contenidos de conciencia" [Bewußtseininhalte] (ALBRECHT, 1997), en la que se incorpora parte del conocimiento del mundo (WOTJAK, 1997). De hecho, en el marco de la semántica de los prototipos - principal representante de este "nuevo comienzo" de la lingüística-, Rosch define el significado en términos de categorías; estas, a su vez, se conciben como clases heterogéneas y no discretas, en las que hay miembros más representativos que otros. El miembro más representativo de una categoría es el prototipo (LAKOFF, 1990; KLEIBER, 2004; CUENCA; HILFERTY, 2011).

Partiendo de la asunción de que "the referential level of semantic structure is part and parcel of a proper semantic description", Geeraerts (2001, p. 8) sostiene que no sólo es admisible, sino también esperable, que el referente "aparezca" en las descripciones de los diccionarios. Así pues, el "prototipo" o, mejor dicho, el "efecto de prototipicidad" [prototypicality effect $]$ puede estar representado de dos maneras distintas en las paráfrasis que se orientan (por lo menos idealmente) hacia un modelo semántico cognitivo: mediante la indicación de los miembros más prototípicos de la categoría, o mediante la identificación de los rasgos más típicos que contribuyen a caracterizar la categoría (GEERAERTS, 2001,p. 8-11). En el primer caso, nos encontramos con un tipo de definición-llamada definición ostensiva- que remite directamente al referente extralingüístico y, por tanto, no está formulada intensionalmente, sino más bien extensionalmente. A este tipo de definición le falta su contraparte intensional ${ }^{6}$. En el segundo caso, por el contrario, las definiciones estarán formuladas intensionalmente ${ }^{7}$.

\footnotetext{
"Por ejemplo: human being "A human being is a man, woman, or child" (CCLDe, 2003, s.v., subrayado original).

${ }^{7}$ Por ejemplo: cabrilla "Pez marino teleósteo, parecido al mero, aunque bastante más pequeño, muy voraz, de color amarillo rosado y de carne fofa e insípida” (DILE, 1997, S.V., ac. 2).
} 
Coseriu (1997, p. 86) critica vehementemente la llamada "semántica cognitiva", pues "como 'cognitiva' no es 'semántica' -no concierne a los significados, sino a las 'cosas'-; y como 'semántica', no es 'cognitiva': no concierne al conocimiento propiamente lingüístico". La crítica se extiende además a la concepción de significado que se engendra en el ámbito de los modelos semánticos cognitivos, en especial la semántica de los prototipos y la semántica de los estereotipos (sobre ésta última, cf. PUTNAM, 1990; HARRAS, 1991, p. 27-45). Coseriu (1997, p. 85) considera que

[L]os "prototipos", "estereotipos", etc. de la (mal) llamada "semántica cognitiva" no son significados son objetos esquemáticos que pueden, a lo sumo, corresponder a ciertos significados, como ejemplos de la realización de tales y cuales "modos de ser" en tales y cuales “entes". Esto debería saberse [...]; pero la (mal) llamada "semántica cognitiva" todavía no se ha enterado de ello.

Sin embargo, Coseriu no está totalmente en desacuerdo con dicho enfoque; lo que hace en realidad es demostrar su rechazo a "las pretensiones de una corriente que se presentaba como la verdadera semántica léxica, a la que creía reemplazar, en vez de entenderse como disciplina complementaria de ella" (ALBRECHT, 2003, p. 52).

Aunque defendemos que sí se debe distinguir entre lenguaje y mundo en el plano estrictamente lingüístico $-\mathrm{y}$ en eso coincidimos con Coseriu, quien basa toda su teoría del lenguaje justamente en esa asunción-, nos vemos obligados a aceptar que una separación estricta entre lengua y realidad extralingüística en la descripción lexicográfica del significado no siempre es factible, y, aun cuando lo es, no necesariamente será provechosa para el usuario ${ }^{8}$.

Desde un punto de vista estrictamente lingüístico, es evidente que lengua y realidad son cosas distintas $-\mathrm{y}$ lo prueba justamente el fenómeno del anisomorfismo interlingüístico (cf. Notas 4 y 5 para algunos ejemplos). Ahora bien, al analizar el problema de la descripción del significado en el ámbito de la lingüística posterior

${ }^{8}$ Sobre los aportes y límites de los modelos estructural y cognitivo al problema de la definición lexicográfica, cf. Bugueño Miranda y Farias (2013a, 2013b) y Farias (2013). 
al llamado "viraje cognoscitivo", Albrecht (2003, p. 52) subraya que "la distinción entre 'mundo' y 'lenguaje' adquiere nuevo sentido". No obstante, aún se echa de menos un análisis sistemático de la manera cómo el conocimiento de las cosas -que se presume universal y compartido entre los interlocutores- se introduce en la técnica del hablar. Se le asigna, a su vez, a la denominada "lingüística esqueuológica" la tarea de investigar la relación entre el "conocimiento de las cosas" y la "configuración y funcionamiento del lenguaje" (ALBRECHT, 2003, p. 42-43). Según Albrecht (2003) parte de la tarea que propone Coseriu en el ámbito de la "lingüística esqueuológica" la realiza justamente la lingüística cognitiva -aunque jamás en el mismo sentido-, lo que no representa una contradicción, si se abandona la idea de una semántica cognitiva absolutamente reaccionaria a los modelos formal y estructural, pasando a entenderla como una disciplina complementaria de este último (ALBRECHT, 1997). El "pero", por así decirlo, de los modelos semánticos cognitivos -y aquí volvemos a la crítica de Coseriu (1997)- estriba justamente en su comprensión de significado, que equivaldría más bien -aunque no completamente- a la noción de concepto.

Desde el punto de vista estrictamente lexicográfico, por otra parte, Geeraerts (2001, p. 14) asevera que "[W]ithin a structuralist conception of semantics, this [sc. el empleo de elementos de carácter extralingüístico en las paráfrasis definidoras] would be inadmissible, because these elements belong to the 'encyclopedic' level rather than the semantic level". La inserción de elementos de carácter extralingüístico en las definiciones se hace posible al adoptarse un modelo semántico cognitivamente orientado como subsidiario del quehacer lexicográfico, según lo expuesto ad supra. Sin embargo, no significa en absoluto borrar los límites entre lengua y realidad. En primer lugar, hay una diferencia clara entre significado definido desde el punto de vista de una teoría lingüística (entidad abstracta) y su expresión mediante una paráfrasis en el diccionario (formulación concreta). En segundo lugar, en el diccionario se trata de aclararle una determinada significación al usuario -que la mayoría de las veces no es especialista en lingüística y sólo quiere resolver sus dudas de manera rápida y objetiva-; la paráfrasis, pues, tiene que redactarse de la manera más simple y clara posible -aunque ello signifique, muchas veces, echar mano de elementos no estrictamente - lingüísticos. 


\section{Las palabras que designan sentimientos/emociones y su descripción lexicográfica}

En la literatura especializada abundan estudios que tratan las palabras que designan sentimientos/emociones ${ }^{9}$ bajo las más distintas perspectivas: la relación que se establece entre lengua, fenómeno psíquico y mundo (por ejemplo, EKMAN, 1992; LINDQUIST et al., 2006; FONTAINE et al., 2007; FRIEDENBERG; SILVERMAN, 2012); la delimitación y descripción del contenido semántico de palabras que designan sentimientos/emociones (por ejemplo, WIERZBICKA, 1992a, 1992b; KÖVECSES, 2008); su tratamiento lexicográfico (por ejemplo, JÄGER; PLUM, 1989; PLUM, 1992; OSTERMANN, 2012). La multitud de trabajos sobre el problema de las palabras que designan sentimientos/ emociones -que aquí sólo puede indicarse muy sumariamente- pone de relieve la complejidad inherente al tema.

Al describir el comportamiento sintáctico-semántico de las palabras que designan sentimientos/emociones, Kailuweit (2009, p. 278) señala que:

L'obiettivo della descrizione linguistica non può essere la spiegazione di processi neurologici. I dati linguistici non danno acceso a questi processi. La descrizione lingüística è un lavoro interpretativo. Essa interpreta dati linguistici da una certa prospettiva e con una certa finalità.

\footnotetext{
${ }^{9}$ Plum (1992) emplea los términos Emotion [emoción] y Gefühl [sentimiento] como sinónimos, siguiendo, pues, una tendencia generalizada en su uso común, fuera del ámbito especializado. La explicación del proceso emocional en el marco de la Psicología de la Emoción, sin embargo, requiere una precisión terminológica. Debe entenderse una emoción como una reacción neurológica -en un nivel inconsciente- ante un estímulo, que se manifiesta mediante un conjunto complejo de activaciones neuronales en el cerebro y se refleja en el cuerpo como un estado emocional externamente observable. Un sentimiento en su estado latente es, a su vez, la experiencia -todavía inconscientede ese estado corporal, que emerge posteriormente cuando el organismo se percata de que su representación del propio estado corporal se ha alterado por la ocurrencia del estímulo, convirtiéndose en un hecho consciente (DAMASIO, 1999, apud BOSSE; JONKER; TREUR, 2008, p. 94-95). Se advierte, pues, una relación estrecha entre ambos conceptos, lo que explica el empleo de los términos emoción y sentimiento como sinónimos en contextos no especializados.
} 
Coincidimos con Kailuweit (2009) en que se debe disociar los conceptos de sentimientos/emociones de los respectivos fenómenos psíquicos, o, en otros términos, en que se debe disociar lenguaje y realidad extralingüística. Ahora bien, y según se ha expuesto en el apartado anterior, consideramos "dass die Abgrenzung unterschiedlichen Wissens in Wörterbüchern nicht nur schwer durchführbar ist, sondern häufig sogar unmöglich ist", teniendo en cuenta "dass es viele Sorten von Wissen gibt, die sowohl Sprach- als auch Weltwissen sind" (BOAS, 2013, p. 92). Eso se refleja claramente en la definición de sustantivos que designan sentimientos/ emociones en diccionarios de lengua. Con el propósito de demostrarlo, se han analizado las definiciones de sustantivos que designan sentimientos/ emociones (Cuadro 1) en los más prestigiados diccionarios de las tradiciones hispánica (DRAEe, 2001; DUEe, 2001; DEA, 2011), portuguesa (AuE, 2009; HouE, 2009; MiE, 1998), italiana (ZVLI, 2011; VTLIe, 1998; DGLI, 2012) y francesa (PRobE, 2001; LLiDF, 1863-1877; DHaLF, 2012).

Cuadro 1 -Artículos léxicos de sustantivos que designan sentimientos/ emociones

\begin{tabular}{|l|l|l|l|}
\hline amor & amor & amore & amour \\
\hline pasión & paixão & passione & passion \\
\hline alegría & alegria & allegria & gaieté \\
\hline gozo & gozo & gioia & joie \\
\hline tristeza & tristeza & tristezza & tristesse \\
\hline melancolía & melancolia & malinconia & mélancolie \\
\hline angustia & angústia & angoscia & angoisse \\
\hline aflicción & aflição & afflizione & affliction \\
\hline odio & ódio & odio & haine \\
\hline cólera & cólera & collera & colère \\
\hline rabia & raiva & rabbia & rage \\
\hline furia & fúria & furia & furie \\
\hline ira & ira & ira & fureur / ire \\
\hline celo(s) & ciúme(s) & gelosia & jalousie \\
\hline envidia & inveja & invidia & envie \\
\hline espanto & espanto & spavento & stupéfaction \\
\hline asombro & assombro & stupore & stupeur \\
\hline miedo & medo & paura & peur \\
\hline temor & temor & timore & crainte \\
\hline
\end{tabular}

Fuente: Autora 
En la Tabla 1 se presenta una síntesis del análisis cuantitativo de los datos. Se ha tratado de identificar la(s) estrategia(s) de definición empleada(s) en cada caso, buscando establecer una relación con el contenido semántico de la unidad léxica en cuestión.

Tabla 1 - Análisis cuantitativo de los datos

\begin{tabular}{|c|c|c|c|c|c|c|c|}
\hline & \multicolumn{6}{|c|}{ Estrategias definitorias } & \multirow{2}{*}{$\begin{array}{l}\text { Total de } \\
\text { definicio- } \\
\text { nes }\end{array}$} \\
\hline & $\begin{array}{l}\text { Defi- } \\
\text { nición } \\
\text { propia }\end{array}$ & $\begin{array}{c}\text { Defi- } \\
\text { nición } \\
\text { impropia }\end{array}$ & $\begin{array}{l}\text { Defini- } \\
\text { ción por } \\
\text { sinóni- } \\
\text { mo(s) }\end{array}$ & \begin{tabular}{|c|} 
Definición \\
parafrástica \\
+ sinóni- \\
$\mathrm{mo}(\mathrm{s})$
\end{tabular} & $\begin{array}{c}\text { Defi- } \\
\text { nición } \\
\text { "mixta" }\end{array}$ & $\begin{array}{c}\text { Defini- } \\
\text { ción con } \\
\text { dos acep- } \\
\text { ciones }\end{array}$ & \\
\hline $\begin{array}{c}\text { Diccio- } \\
\text { narios de } \\
\text { español }\end{array}$ & 57 & 3 & 12 & - & 10 & 1 & 83 \\
\hline $\begin{array}{c}\text { Diccio- } \\
\text { narios de } \\
\text { portugués }\end{array}$ & 44 & 1 & 23 & 31 & - & 3 & 102 \\
\hline $\begin{array}{c}\text { Diccio- } \\
\text { narios de } \\
\text { italiano }\end{array}$ & 43 & 7 & 3 & 8 & 1 & 4 & 66 \\
\hline $\begin{array}{l}\text { Diccio- } \\
\text { narios de } \\
\text { francés }\end{array}$ & 65 & 3 & 5 & 3 & - & 7 & 83 \\
\hline
\end{tabular}

Fuente: Autora

El número total de definiciones corresponde al conjunto de todas las definiciones recolectadas en los artículos de cada una de las unidades léxicas seleccionadas en los tres diccionarios de cada lengua que se han analizado $^{10}$. El análisis permitió identificar el empleo de seis mecanismos explicativos distintos:

a. Definición propia: Es la definición en metalenguaje de contenido (cf. SECO, 2003). La paráfrasis presenta un pattern sintáctico que se acerca al modelo genus proximum et differentia(e) specifica(e) -aunque no son definiciones de este tipo en sentido estricto, o por lo menos no desde el punto de vista de su aplicación en el marco de un modelo semántico

\footnotetext{
${ }^{10}$ Se han considerado para el análisis las "paráfrasis" relativas al sentimiento/a la emoción en cuestión; en muchos artículos, sin embargo, había más de una definición para el sentimiento/emoción.
} 
estructural (cf. resultados del análisis cualitativo ad infra). Por ejemplo: ciúme "Inquietação mental causada por suspeita ou receio de rivalidade no amor ou em outra aspiração" (MiE, 1998, s.v., ac.1).

b. Definición impropia: Es la definición en metalenguaje de signo (cf. SECO, 2003). La paráfrasis presenta un pattern sintáctico que imposibilita la aplicación de la prueba de sustitución" " Por ejemplo: paixão "Mais comumente paixão designa amor, atração de um sexo pelo outro" (MiE, 1998, s.v., ac.3).

c. Definición por sinónimo(s): Se presentan tan sólo "sinónimos" del signo-lema, y no una paráfrasis stricto sensu. Por ejemplo: aflicción "Pesadumbre o tristeza" (DEA, 2011, s.v., ac.1).

d. Definición parafrástica + sinónimos: Se presenta una definición -en general, en metalenguaje de contenido- $\mathrm{y}$, a continuación, uno o más "sinónimos" del signo-lema. Por ejemplo: gioia "sentimento di piena e viva soddisfazione dell'animo; allegria, felicità" (DGLI, 2012, s.v., ac.1).

e. Definición "mixta": Se trata de una paráfrasis "bipartita": la primera parte corresponde a una definición en metalenguaje de contenido; la segunda concierne a una definición en metalenguaje de signo. Por ejemplo: allegria "Contentezza, buon umore, anche di più persone insieme, che si esterna in modo vivace e spesso rumoroso; differisce dall'allegrezza in quanto, più che il sentimento, ne è l'aspetto e la manifestazione esteriore, e può anche essere uno stato abituale" (VTLIe, 1998, s.v., ac.1).

f. Definición con dos acepciones: Al igual que en el caso anterior, es una suerte de paráfrasis "bipartita"; cada parte corresponde a la definición de una significación distinta. Por ejemplo: jalousie "Sentiment hostile qu'on éprouve en voyant un autre jouir d'un avantage qu'on ne possède pas ou qu'on désirerait posséder exclusivement ; inquiétude qu'inspire la crainte de partager cet avantage ou de le perdre au profit d'autrui"' (PRobE, 2001, s.v., ac.2).

${ }^{11}$ Para una discusión en torno de la importancia y los límites de la prueba de la sustitución en el ámbito lexicográfico, cf. Seco (2003). 
Se han desconsiderado, ante todo, las definiciones "mixtas" y las definiciones con dos acepciones, porque, en ambos casos, se trata más bien de errores metodológicos (sobre el particular, cf. BUGUEÑO MIRANDA; FARIAS, 2009). Con relación al material analizado, aunque los diccionarios, de una manera general, han mostrado una marcada tendencia al empleo de definiciones propias, llama la atención también la diversidad de estrategias de definición que se mezclan o se combinan con las paráfrasis en metalenguaje de contenido en las obras mencionadas $a d$ supra $^{12}$. Ello es un fuerte indicio de que sí se reconoce los sustantivos que designan sentimientos/emociones como unidades léxicas potencialmente difíciles de definir -al menos si se trata de definirlos de acuerdo con el modelo genus proximum et differentia(e) specifica(e)-.

El análisis cualitativo de los datos ha considerado solamente las definiciones propias. Partiéndose de los resultados obtenidos en el análisis cuantitativo, se asume que las definiciones propias -aunque, desde el punto de vista sintáctico, toman la forma de genus proximum et differentia(e) specifica(e)-, desde el punto de vista "semántico", por así decirlo, no corresponden a un modelo formal/estructural, puesto que no se limitan a la enumeración de rasgos característicos/semas de la entidad/unidad léxica definida. Así pues, se ha tratado de identificar los tipos de informaciones que componen las definiciones de sustantivos que designan sentimientos/ emociones. Los principales tipos de definiciones son los siguientes:

a. Definición por remisión a otros sentimientos/otras emociones: Desde el punto de vista metodológico, esta estrategia se acerca a la definición por sinónimo(s). Así pues, aunque no se trata de un error metodológico stricto sensu -analógicamente, las definiciones por sinónimo(s) son muchas veces más aclaradoras que una definición parafrástica (BUGUEÑO MIRANDA; FARIAS, 2011c)-, tampoco se puede soslayar el hecho de que definiciones de este tipo suelen conllevar consecuencias peligrosas. En primer lugar, es posible que, así como en el caso de las definiciones por sinónimo(s), el

\footnotetext{
${ }^{12} \mathrm{El}$ foco aquí es el análisis de las estrategias empleadas en la definición de los sustantivos que designan sentimientos/emociones, sin la preocupación por establecer una comparación entre los distintos diccionarios/las distintas tradiciones lexicográficas. Ello, claro está, encontraría su legitimación en otra ocasión.
} 
usuario necesite realizar una serie de consultas consecutivas al diccionario hasta poder aclarar el significado de una unidad léxica. En segundo lugar, y aún más grave, siempre existe la posibilidad de que se genere un "círculo vicioso" en la definición (MARTÍNEZ DE SOUZA, 1995, s.v. definición lingüística). Ejemplos: alegria "Sentimento de felicidade, de contentamento, satisfação, júbilo" (AuE, 2009, s.v., ac.3); crainte "Sentiment de peur, d'inquiétude" (DHaLF, 2012, s.v.); passione "Violento sentimento di amore o desiderio sensuale" (ZVLI, 2011, s.v., ac.5).

b. Definición por referencia a causas/manifestaciones del sentimiento/la emoción: Las definiciones de este tipo corresponden a descripciones, la mayoría de las veces relativamente largas, de las causas que desencadenan el sentimiento/la emoción en cuestión y/o de las reacciones que el sentimiento/la emoción provocan en quienes 1o/la experimentan. Ejemplos: alegría "Sentimiento que produce en alguien un suceso favorable o la obtención de algo que deseaba o que satisface sus sentimientos o afectos" (DUEe, 2001, s.v., ac.1); haine "Sentiment violent qui pousse à vouloir du mal à qqn et à se réjouir du mal qui lui arrive" (PRobE, 2001, s.V., ac.1); ira "Sentimento per lo più improvviso e violento, che, provocato dal comportamento di persone o da fatti, circostanze, avvenimenti, tende a sfogarsi con parole concitate, talvolta con offese, con atti di rabbia e di risentimento, con una punizione eccessiva o con la vendetta, contro chi, volontariamente o involontariamente, lo ha provocato" (VTLIe, 1998, s.v.).

La síntesis del análisis cualitativo de los datos es la siguiente:

a. Se reafirma la asunción generalmente aceptada según la cual el contenido de las paráfrasis de sustantivos que designan sentimientos/emociones no puede corresponder a un conjunto de condiciones necesarias y suficientes (JÄGER; PLUM, 1989; PLUM, 1992; WIERZBICKA, 1992b).

b. Se confirma la hipótesis de que las paráfrasis de sustantivos que designan sentimientos/emociones deberían basarse en 
constructos estereotipados de las "causas que provocan el sentimiento/la emoción" [emotionserzeugenden Anlässen], así como de las "formas convencionalizadas de conductas emocionales" [konvetionalisierten Formen emotionalen Benehmens] (PLUM, 1992, p. 12). Sin embargo, aquí surgen dos cuestiones: i) cómo debería hacerse la selección de las informaciones que entrarían a componer las paráfrasis en cada caso -en otros términos, qué criterios validarían su estatuto de "constructos estereotipados (o prototípicos) de las causas/manifestaciones de los sentimientos/las emociones en cuestión-; y ii) cuál es el real poder explicativo de las paráfrasis así formuladas.

\section{Los aportes de dos modelos semánticos a la definición de sentimientos/emociones}

$\mathrm{Si}$, por una parte, el análisis que se llevó a cabo demostró la imposibilidad de definición de los sustantivos que designan sentimientos/ emociones dentro del marco de una semántica formal/estructural, por otra, también puso de manifiesto la carencia, en el ámbito (meta)lexicográfico, de un modelo mínimamente consecuente para su definición. Lo único que se ha sacado en claro hasta el momento es que, de hecho, hay que incorporar informaciones extralingüísticas en las paráfrasis de este conjunto de unidades léxicas. Así pues, considerando que la apreciación crítica de las teorías semánticas es el primer paso hacia el desarrollo de una propuesta para el perfeccionamiento de las definiciones de sustantivos que designan sentimientos/emociones en los diccionarios de lengua, se evaluarán, a continuación, los posibles aportes de dos modelos semánticos postestructurales al problema que se ha presentado, buscando respuestas a las cuestiones propuestas como cierre del apartado anterior.

Entre los modelos postestructurales ${ }^{13}$, la semántica cognitiva-con especial referencia a la teoría de los prototipos-ocupa, sin lugar a dudas, una posición de destaque. No se la considerará en este trabajo por dos razones. En primer lugar, porque a lo largo de los últimos treinta años ya se ha propuesto la aplicación de la semántica prototípica en la praxis

\footnotetext{
${ }^{13}$ Para un panorama general de los modelos semánticos postestructurales, cf. Geeraerts (2010).
} 
lexicográfica, como modelo alternativo o complementario a la semántica estructural. Sin embargo, los resultados obtenidos con la aplicación de la semántica prototípica a la generación de las definiciones, por ejemplo, no siempre logran suplantar los resultados obtenidos con los modelos formal/estructural ${ }^{14}$-aunque, claro está, la concepción de significado que se engendra en ese ámbito ha tenido (y sigue teniendo) su importancia en el quehacer lexicográfico (BUGUEÑO MIRANDA; FARIAS, 2013a, 2013b)-. En segundo lugar, el modelo cognitivo -al contrario de los modelos formal y estructural, con todas las limitaciones que se les pueda asignar- no ofrece una metodología que permita acceder al significado de las unidades léxicas. La delimitación de los rasgos característicos/ semas constituye, de hecho, una "herramienta" metodológica-altamente satisfactoria intensionalmente, aunque, por cierto, frágil desde el punto de vista extensional (KLEIBER, 2004, p. 31-37) - que permite expresar en términos lingüísticos la abstracción en que consiste al fin y al cabo el significado. En cambio, la semántica prototípica no nos ofrece una metodología objetiva que nos permita deslindar los elementos pertinentes en la descripción lexicográfica del significado: la selección de los elementos que entrarán a componer la definición con base en el prototipo no deja de ser meramente impresionista; ello, al menos en parte, se debe al hecho de que la prototipicidad también es culturalmente determinada, una vez que, de acuerdo con Rosch (1978), la categorización se basa en experiencias colectivas 0 , incluso, personales ${ }^{15}$.

\footnotetext{
${ }^{14}$ Según Farias (2013, p. 141-149), hay tres aspectos directamente involucrados en el proceso de definir: la discriminación de las acepciones, la ordenación de las acepciones y la formulación de las paráfrasis. Cuando se afirma que los resultados obtenidos con la aplicación de la semántica prototípica no siempre logran suplantar los resultados obtenidos con los modelos formal/estructural, ello se refiere específicamente al tercer aspecto -que es justamente el aspecto que nos interesa aquí. Sobre el particular, cf., por ejemplo, los resultados publicados en Bugueño Miranda y Farias (2011a, 2013a, 2013b) y Farias (2013). En lo que concierne a los demás aspectos -que, a su vez, están estrechamente ligados al problema de la polisemia- sobresalen las contribuciones de la semántica cognitiva. Sobre las contribuciones de la semántica cognitiva al problema de la polisemia desde el punto de vista lexicográfico, cf., por ejemplo, Geeraerts (1990, 2001, 2003, 2007), Kilgarriff (1997, 2007), Gao (2013), Oliveira; Bugueño Miranda; Siqueira (2013).

${ }^{15}$ Sobre el particular, García Márquez, en su crónica "La vaina de los diccionarios", publicada en el periódico El País en 19 de mayo de 1982, ofrece un ejemplo bastante
} 
En lo que atañe a los demás modelos semánticos postestructurales, se considerarán tan sólo el metalenguaje semántico natural y la semántica de marcos. Ambos modelos, aunque diametralmente opuestos en su esencia -el primero se encuentra enmarcado dentro del llamado "neoestructuralismo", mientras que el segundo se ha engendrado en el seno de la lingüística cognitiva-, se acercan en lo que concierne a su orientación lexicográfica.

\section{El metalenguaje semántico natural}

La idea de definir el significado de una palabra por medio de conceptos "primitivos" -es decir, conceptos que se presume sean "indefinibles"- no es precisamente novedosa en el ámbito del estructuralismo. Sobre esa base, Wierzbicka propone, en los comienzos de los años 1970, un modelo de análisis del significado -denominado metalenguaje semántico natural- que se ha desarrollado y perfeccionado a lo largo de las décadas siguientes (WIERZBICKA, 1985, 1992a, 1992b, 1999; GODDARD, 2010). Geeraerts (2010, p. 130) aclara que la diferencia entre el modelo de análisis componencial propuesto primeramente en Pottier (1977) y el metalenguaje semántico natural estriba en que mientras el primero presenta una descripción típicamente referencial de las unidades léxicas pertenecientes a una determinada categoría (el ejemplo clásico es la categoría "asiento"), el segundo toma en consideración asimismo la percepción del hablante acerca de lo referido -en otros términos, la dimensión cognitiva. Por ello es que se considera el metalenguaje semántico natural un modelo cognitivamente orientado (sobre el particular, cf. GODDARD, 2010).

El modelo de Wierzbicka se basa sobre dos pilares fundamentales: (a) la existencia -y consecuente posibilidad de delimitación- de primitivos/universales semánticos y (b) la propuesta de explicación del significado de las unidades léxicas mediante una paráfrasis reductora.

\footnotetext{
interesante: "Otra cosa que me inquietó siempre del diccionario de la Real Academia es la definición de los colores. Amarillo: 'Del color semejante al del oro, el limón, la flor de la retama, etcétera’. A mi modo de ver las cosas desde la América Latina, el oro era dorado, no conocía las flores de la retama, y el limón no era amarillo, sino verde. [...] Necesité muchos años para viajar a Europa y darme cuenta de que el diccionario tenía razón, porque, en realidad, los limones europeos son amarillos".
} 
La asunción básica que fundamenta el metalenguaje semántico natural es que las definiciones no deberían estar redactadas en un lenguaje técnico -es decir, mediante un sistema representacional formalizado-, sino en un lenguaje natural. Así pues, el objetivo consiste en identificar y catalogar los llamados "primitivos/universales semánticos", entendidos -según la hipótesis fuerte de lexicalizacióncomo conceptos universalmente lexicalizados, que contrastan, por tanto, con los conceptos culturalmente delimitados. Ello significa decir $-\mathrm{y}$ suponer-que hay conceptos básicos que se pueden expresar en todas las lenguas mediante una palabra o expresión específica. El metalenguaje semántico natural, hasta el momento, ha identificado alrededor de 60 conceptos universales; sin embargo, el número aumenta cada año con la inclusión de nuevos conceptos en la lista. Ejemplos de conceptos universales son: " $P$ ", "you", "someone/person", "something", "thing" (sustantivos); "kind", "part" (sustantivos relacionales); "good", "bad" (evaluadores); "big", "small" (descriptores), etc. Se ha podido probar la existencia de unidades lexicalizadas para expresar la mayor parte de estos conceptos en una amplia variedad de idiomas; dentro del marco del metalenguaje semántico natural, ello contribuiría a comprobar su universalidad (GEERAERTS, 2010, p. 128-129).

Las paráfrasis reductoras, a su vez, se formulan mediante universales semánticos. La definición, pues, corresponde a una suerte de desglose de un concepto no primitivo en conceptos primitivos. Así pues, la descripción semántica de nombres que designan sentimientos/ emociones se lleva a cabo mediante primitivos semánticos, como "want", "say", "think", "know", "good", "bad", etc. (WIERZBICKA, 1992b, p. 541-542). Sobre esa base, Wierzbicka (1999) presenta un modelo estandarizado de descripción semántica de sustantivos que designan sentimientos/emociones. Como ejemplo citamos aquí el sustantivo afraid [miedo]:

\author{
I felt afraid. = \\ I felt something because I thought something \\ sometimes a person thinks: \\ "something bad can happen to me now \\ I don't want this to happen \\ because of this I want to do something \\ I don't know what I can do"
}


because this person thinks this, this person feels something bad

I felt (something) like this because I thought something like this

(WIERZBICKA, 1999, p. 14)

Se trata de una suerte de descripción prototípicamente orientada, es decir, una descripción de un tipo de situación -denominada escenario- que está asociada a un sentimiento/una emoción en la mente de las personas. El escenario -como constructo prototípico- está, pues, cognitivamente determinado (WIERZBICKA, 1999, p. 12-17).

Basado en la hipótesis del relativismo lingüístico de Sapir-Whorf, Wierzbicka y sus discípulos cuestionan la posibilidad de delimitación de un conjunto de "emociones básicas, tal como se lo ha planteado, muchas veces, en el ámbito de la Psicología de la Emoción. Frente a la propuesta de delimitación de diez emociones básicas -entendidas, por tanto, como universales-, lexicalizadas en inglés como interest, joy, surprise, sadness, anger, disgust, contempt, fear, shame/shyness y guilt, Wierzbicka (1992a, p. 119) reacciona: "English terms of emotion constitute a folk taxonomy, not an objective, culture-free analytical framework". Así pues, por un lado, el polonés no presenta un equivalente perfecto para el inglés disgust; por otro, una de las lenguas aborígenes de Australia no establece una distinción, a nivel léxico, entre fear y shame. Lindquist et al. (2006) también comparten la opinión de Wierzbicka, al afirmar que "people of different cultures divide the affective world into different basic emotion categories $[\ldots]$, such that emotion concepts differ across cultures".

Ahora bien, la imposibilidad de delimitar emociones básicas no impediría la definición mediante universales semánticos. Wierzbicka (1999, p. 15-16) explica que:

First, all languages have a general, undifferentiated word for FEEL (covering both thought-related and nonthought-related kinds of feelings), and that, second, all languages have some words for some particular kinds of thought-related feelings (e.g. afraid and guilty in English and toska in Russian). The meanings of such words are language-specific and, generally speaking, do not match across languages and cultures. Every language, however, 
has lexically encoded some scenarios involving both thoughts and feelings and serving as a reference point for the identification of what the speakers of this language see as distinct kinds of feelings. For example, Russian has no word for guilt, and of course English has no word for toska; but both guilt and toska identify what the speakers of the language see as a specific kind of feeling, associated with an identifiable cognitive scenario.

Since the cognitive scenarios linked with guilt and toska can be stated in the same, universal human concepts (such as FEEL, WANT, BAD, DO, and so on), these scenarios can be understood by cultural outsiders, and the kinds of feeling associated whit them can be identified, explained, and compared; and both the similarities and differences between scenarios lexicalized in different languages can be pinpointed. But the very possibility of comparisons rests on the availability of a universal tertium comparationis, provided by the universal concepts like FEEL, WANT, BAD, GOOD, or DO, and universally available configurations of concepts such as, for example, "I feel like this".

En el ámbito (meta)lexicográfico, la posibilidad de identificación de un conjunto de "instancias prototípicas" relacionadas con un determinado concepto constituye el principal aporte del metalenguaje semántico natural. Así pues, las llamadas "instancias prototípicas" pueden contribuir en la definición de sentimientos/emociones -o, mejor dicho, de "estados emocionales"-, al proponer que sus rasgos conceptuales fundamentales serían la causa y el prototipo situacional ${ }^{16}$. Sin embargo, se advierte que "different emotion concepts have different structures and that some of them specify certain thoughts as the causes of the emotional state, whereas others do not" (WIERZBICKA, 1992a, p. 177) ${ }^{17}$.

Por un lado, esa propuesta representa un avance frente a los modelos formal/estructural, pues ofrece la posibilidad de inserción de elementos extralingüísticos en las paráfrasis. Por otro, es posible advertir un avance asimismo en comparación con la semántica prototípica, ya que

\footnotetext{
${ }^{16} \mathrm{Cf}$. el esquema conceptual de afraid presentado ad supra.

${ }^{17} \mathrm{Se}$ sabe, por ejemplo, con base en la experiencia del mundo, que la mayoría de las veces no se puede determinar el origen del sentimiento de melancolía; así pues, en una definición de melancolía, no se explicitaría la causa del estado emocional.
} 
existe una propuesta metodológica que permite seleccionar de manera más objetiva los rasgos extralingüísticos que deberán ayudar a generar las paráfrasis.

Para finalizar, se mencionan sumariamente los límites de este modelo teórico, que le restan fuerza para una descripción lexicográfica del significado del todo satisfactoria. En lo concerniente al primer fundamento, Geeraerts (2010, p. 133-134) destaca, en primer lugar, que la asunción básica según la cual una definición mediante universales semánticos sería más inteligible que una paráfrasis "tradicional" es problemática. Las definiciones no son sometidas sistemáticamente a la evaluación de los usuarios de diccionarios, quienes podrían juzgar con propiedad la superioridad del metalenguaje semántico natural frente al modelo genus proximum et differentia(e) specifica(e), por ejemplo. La inteligibilidad de una definición, al fin y al cabo, depende menos de una formulación por medio de "universales semánticos" que de una formulación a partir de elementos previamente conocidos por los usuarios. En segundo lugar, la hipótesis fuerte de lexicalización de Goddard plantea que la identificación de los universales semánticos depende de la comparación de un conjunto -en principio, exhaustivo- de lenguas. Sin embargo, aún quedan dudas respecto al postulado de "lexicalización universal". Por un lado, hay conceptos -entre los que hasta ahora se aceptan como universales- que no están lexicalizados en determinados idiomas - por ejemplo, BEFORE y AFTER en maya yucateco (BOHNEMEYER, 2003, apud GEERAERTS, 2010, p. 133)-. Por otro lado, la comparación de las lenguas a partir del significado de las distintas expresiones lexicalizadas en cada idioma plantea dos problemas: (a) desde el punto de vista lingüístico, aceptar la existencia de una suerte de "equivalencia conceptual" entre las formas lexicalizadas correspondientes a los universales en cada lengua es ignorar el hecho elemental del anisomorfismo interlingüístico; (b) desde el punto de vista metodológico, establecer una comparación en el plano conceptual supone la posibilidad de generar paráfrasis para describir el significado de los universales; sin embargo, esto hiere uno de los principios básicos del modelo, puesto que los universales serían -por antonomasia-indefinibles.

Geeraerts (2010, p. 135-137) discute también problemas concernientes al segundo fundamento del metalenguaje semántico natural. Como se sabe, en los modelos parafrásticos cuya formulación consiste en explicitar un conjunto de rasgos, los rasgos que componen la paráfrasis deben funcionar como una suerte de "lista de chequeo", que se 
aplique a la realidad extralingüística, permitiendo, a la vez, caracterizar una determinada entidad y contrastarla con otras. Ahora bien, no siempre los universales identificados al interior de una dada "instancia prototípica" y que, teóricamente, deberían emplearse en la generación de la definición son "necesarios y suficientes" para caracterizar la entidad extralingüística, oponiéndola a otras. A modo de ilustración, Geeraerts (2010) cita la propuesta de Wierzbicka (1985, p. 299-300) para la definición de fruit. Según la autora, entre los rasgos que caracterizan la entidad en cuestión están": (1) "poseer una cubierta exterior más resistente que la parte interior", (2) "poseer partículas pequeñas en su interior, separadas de las demás partes”, (3) "poseer pulpa jugosa”, (4) "ser posible comerla cruda, sin ninguna preparación previa" y (5) "ser bueno para la salud comerla cruda". Sin embargo, es evidente que no todos los rasgos mencionados pueden aplicarse, de manera general, a todos las frutas -el rasgo (1) no se aplica a la fresa, de la misma manera que los rasgos (2) y (3) no se aplican a la banana, por ejemplo. Asimismo, parte de los rasgos -nos referimos específicamente a (4) y (5)- no se aplican exclusivamente a las frutas, sino que podrían aplicarse a algunas legumbres. No obstante, en honor de la verdad, hay que reconocer que Wierzbicka trata, de alguna manera, de defenderse de las críticas, aclarando, con respecto a los sentimientos/emociones, que diferentes conceptos pueden presentar diferentes estructuras. Sin embargo y pese a la honestidad de la autora al reconocer las posibles lagunas de su modelo, su constatación, aunque no invalida por completo el segundo postulado, le resta mucha fuerza.

\section{La semántica de marcos}

La semántica de marcos-como modelo cognitivamente orientadoparte de la base de que "words represent categorizations of experience, and each of these categories is underlain by a motivating situation occurring against a background of knowledge and experience" (FILLMORE, 1982, p. 112). Así pues, se describen los significados de las palabras y sintagmas, teniendo en cuenta no sólo los contextos (lingüísticos) en que se encuentran, sino también el marco (extralingüístico) -o frame- en que están inseridos (ATKINS; RUNDELL, 2008, p. 145).

\footnotetext{
${ }^{18}$ Se reproduce aquí sólo parcialmente y de manera simplificada los rasgos definidos en Wierzbicka (1985).
} 
Aunque Fillmore (1982), de hecho, aclara que hay que establecer una diferencia entre "conocimiento sobre las palabras" y "conocimiento sobre las cosas" -algo que, sea dicho de paso, no suena para nada novedoso-, los postulados generales sobre los que se basa la semántica de marcos evidencian una "comprensión extensional del significado"19. En este sentido, Wegner (1989), por ejemplo, señalaba ya que el conocimiento lingüístico [Sprachwissen] y el conocimiento situacional [Situationswissen] -o enciclopédico/extralingüístico- no son independientes, sino que están en mutua dependencia. Sin embargo, más que reconocer la interdependencia entre conocimiento lingüístico y extralingüístico - lo que ya había quedado claro con la semántica prototípica-, la principal contribución de este modelo teórico reside en el intento de presentar un instrumento que permita acceder al conocimiento extralingüístico pertinente en la descripción lingüística del significado.

El interés de los lexicógrafos en la semántica de marcos no es, pues, algo reciente. Ballmer y Brennenstuhl (1981), por ejemplo, presentan una propuesta de aplicación del concepto de frames -algo entonces completamente novedoso y aun carente de precisiones teóricasa la descripción semántica de los verbos en alemán y su presentación en los diccionarios. Años más tarde, Fillmore y Atkins (1994) plantean que este modelo podría contribuir además a la descripción de la polisemia, de los patrones colocacionales y de la valencia de verbos y nombres en los diccionarios de lengua.

Más específicamente en el ámbito de la descripción del significado, Wegner (1989) defiende la semántica de marcos como una solución alternativa frente al modelo tradicional. Kammerer (1999), a su vez, presenta un modelo de "descripción lexicográfica del significado basada en frames" [framebasierte lexikographischen Bedeutungsbeschreibung]. Según la propuesta del autor, los frames estarían proyectados para almacenar de forma sistemática el conocimiento común y, la mayoría de las veces, estereotipado acerca de los referentes extralingüísticos de las unidades léxicas de una dada lengua; parte de este conocimiento entraría en la formulación de las paráfrasis definidoras.

A pesar del gran interés que la semántica de marcos ha despertado entre los lexicógrafos, hay pocos registros de la aplicación práctica de este

\footnotetext{
${ }^{19}$ Sobre las nociones de "comprensión intensional" y "extensional" del significado, cf. Bugueño Miranda y Farias (2013a; 2013b).
} 
modelo teórico en la elaboración de diccionarios. Los dos únicos casos de que tenemos noticias son el Algemeen Nederlands Woordenboek (ANW) y el Online-Wortschatz-Informationssystem Deutsch (OWID). El ANW -diccionario general del holandés- es un diccionario electrónico de nueva planta, cuya principal contribución estriba, justamente, en la aplicación de los frames a la descripción del significado: las definiciones van acompañadas de estructuras denominadas "semagramas" [semagrams], que, según la definición de los directores del proyecto, son esquemas estructurales abstractos -0 , en términos más simples, frames-, que se llenan con datos lexicales concretos (MOERDIJK, 2008) ${ }^{20}$. El OWID es, a su vez, un diccionario general electrónico del alemán, elaborado por un grupo de investigadores vinculados al Institut für deutsche Sprache en Mannheim. La noción de frames se aplica aquí no sólo a la descripción del significado stricto sensu -en otros términos, a la generación de las paráfrasis definidoras, como en el caso del ANW-, sino también a la descripción detallada del signo-lema tanto en el plano sintagmático (por ejemplo, las colocaciones) como paradigmático (por ejemplo, los sinónimos y antónimos) $)^{21}$.

\section{El proyecto FrameNet y su potencial de aplicación lexico- gráfica}

El proyecto FrameNet se fundamenta en los postulados de la semántica de marcos y su finalidad es la aplicación en la descripción del significado de las unidades léxicas, especialmente en el ámbito lexicográfico (STEINER, 2004).

Los frames se definen, en el ámbito de la FrameNet, como "schematic representations of the conceptual structures and patterns of beliefs, practices, institutions, images, etc., that provide a foundation for meaningful interaction in a given speech community" (FILLMORE et al., 2003, p. 235). El objetivo final es describir "the combinatory potential of a lexical item and indicating explicitly how each "frame element' can be realized, syntactically and lexically, at the surface level" (FONTENELLE, 2012, p. 442).

\footnotetext{
${ }^{20}$ Sobre la concepción del ANW, cf. también Moerdijk et al. (2008) y Farias (2013, p. 262-266).

${ }^{21}$ Sobre la concepción del OWID, cf. Müller-Spitzer y Möhrs (2008) y Klosa et al. (2011).
} 
El proyecto original -que se viene desarrollando desde 1997 en el International Computer Science Institute en Berkeley- tiene como objetivo la construcción de una base de datos del inglés. De forma paralela a la FrameNet del inglés, se están desarrollando actualmente FrameNets para otras lenguas, como el alemán, el español, el francés, el japonés, el búlgaro y el portugués ${ }^{22}$.

En el ámbito lexicográfico, Atkins y Rundell (2008, p. 150159) analizan el empleo de la FrameNet como herramienta auxiliar en la tarea de discriminación de las acepciones en los diccionarios. Boas (2001, 2002), a su vez, enfatiza la posibilidad de aplicación de los datos proporcionados por la FrameNet a la elaboración de diccionarios bilingües inglés-alemán. Por fin, Prieto (2008) presenta una propuesta concreta para la aplicación de la anotación semántica del corpus de la FrameNet a la descripción valencial en un diccionario de colocaciones del español. Sin embargo, se destacarán aquí los resultados de Ostermann (2012) y Farias (2013) en relación a la posibilidad de aplicación de la FrameNet a la generación de definiciones de sustantivos que designan sentimientos/emociones.

Ostermann (2012) elabora una suerte de "estructura cognitiva de definición" [cognitive defining structure] para términos básicos de emociones [basic emotions terms], que se fundamenta en los postulados de la semántica de marcos y su aplicación práctica en la FrameNet, por un lado, y los modelos cognitivos idealizados, por otro. Desde el punto de vista formal, la autora emplea la fórmula genus proximum et differentia(e) specifica(e), siendo que al genus proximum corresponde el hiperónimo feeling [sentimiento] y a la differentia specifica corresponde

${ }^{22}$ Los frames se conciben como estructuras conceptuales esencialmente extralingüísticas, sin conexión con una lengua particular. Así pues, se justifica que las FrameNets que se generan para distintas lenguas puedan asumir como punto de partida la FrameNet del inglés (GODDARD, 2010, p. 80-81). Ahora bien, los estudios más recientes sobre la generación de FrameNets para otras lenguas ponen de manifiesto una serie de problemas que de alguna manera le restan fuerza a la asunción general según la cual los frames serían estructuras universales. Se revela tanto la necesidad de adaptación de algunos frames definidos para el inglés, como de introducción de nuevos frames, debidas en gran medida a los distintos patrones de lexicalización, así como las divergencias entre las estructuras polisémicas de las lenguas. Cf., por ejemplo, Ohara (2012), Koeva (2010) y Salomão (2009). 
una when-definition que presenta la "causa de la emoción” y la "reacción a la emoción".

Cuadro 2 - Esquema de definición de Ostermann (2012) ${ }^{23}$

\begin{tabular}{|l|c|c|c|c|}
\hline \multirow{2}{*}{$\begin{array}{l}\text { NUEVA ESTRUCTURA } \\
\text { COGNITIVA DE } \\
\text { DEFINICIÓN }\end{array}$} & $\begin{array}{c}\text { definiens } \\
\text { (signo-lema) }\end{array}$ & $\begin{array}{c}\text { genus } \\
\text { proximum }\end{array}$ & \multicolumn{2}{|c|}{ differentia specifica } \\
\cline { 2 - 5 } & $\begin{array}{c}\text { sustantivo que } \\
\text { designa sentimento/ } \\
\text { emoción }\end{array}$ & $\begin{array}{c}\text { emoción/ esta- } \\
\text { do emocional }\end{array}$ & causa & reacción \\
\hline
\end{tabular}

Fuente: Ostermann (2012, p. 496)

El esquema que se presenta ad supra constituye la base sobre la que se generan las paráfrasis para los sustantivos que designan sentimientos/emociones en inglés. Por ejemplo:

anger a bad feeling rising in you when you think sth or sb's behaviour is unfair or unjustified and you would immediately like to do sth about it; it might be hard to suppress this wish to do sth, you might act in an unfriendly way, get red in the face or shout (Ostermann, 2012, p. 496, subrayado original).

La principal ventaja de este modelo en comparación con las definiciones "tradicionales", por así decirlo, consiste en la sistematización de las informaciones y la consecuente estandarización de las paráfrasis de una determinada categoría. No obstante, su mayor desventaja es el propio pattern sintáctico propuesto. En primer lugar, se trata de fundir dos métodos de definición a principio incompatibles. En segundo lugar, las paráfrasis resultan muy largas y sintácticamente complejas, presentando una serie de informaciones que no son esenciales en la definición.

El modelo de Ostermann (2012), pese a todas las críticas que se le puedan dirigir, sirve como punto de partida para el desarrollo de la propuesta de Farias (2013). Esa autora presenta un esquema de definición a partir de las informaciones obtenidas mediante el análisis de los frames

${ }^{23}$ Traducido y adaptado del inglés. 
EMOTIONS y FEELING en la FrameNet del inglés ${ }^{24}$, cuyos slots -que podrán llenarse o no con las informaciones del corpus- deben convertirse en los rasgos mínimos de la definición, según se presenta en el Cuadro 3.

Cuadro 3 - Esquema de definición de Farias (2013) ${ }^{25}$

\begin{tabular}{|c|c|c|c|c|c|c|}
\hline \multirow{3}{*}{\begin{tabular}{|l|} 
definiens \\
(signo- \\
lema)
\end{tabular}} & \multicolumn{3}{|c|}{ Hiperónimo } & \multicolumn{3}{|c|}{ Especificadores } \\
\hline & \multicolumn{2}{|c|}{ Término genérico } & \multirow{2}{*}{$\begin{array}{l}\text { (Evalua- } \\
\text { ción) }\end{array}$} & & & \\
\hline & Emoción & $\begin{array}{l}\text { Naturaleza } \\
\text { de la emo- } \\
\text { ción }\end{array}$ & & (Duración) & (Estímulo) & $\begin{array}{l}\text { (Expre- } \\
\text { sión) }\end{array}$ \\
\hline $\begin{array}{l}\text { sustantivo } \\
\text { que desig- } \\
\text { na sen- } \\
\text { timento/ } \\
\text { emoción }\end{array}$ & $\begin{array}{l}\text { senti- } \\
\text { miento }\end{array}$ & $\begin{array}{l}\text { tipo de emo- } \\
\text { ción }\end{array}$ & \begin{tabular}{|c|} 
(juicio \\
de valor \\
acerca de la \\
emoción)
\end{tabular} & $\begin{array}{l}\text { (tran- } \\
\text { sitorio/ } \\
\text { duradero) }\end{array}$ & $\begin{array}{l}\text { (causa del } \\
\text { sentimien- } \\
\text { to) }\end{array}$ & $\begin{array}{l}\text { (signos } \\
\text { prototí- } \\
\text { picos del } \\
\text { sentimien- } \\
\text { to) }\end{array}$ \\
\hline
\end{tabular}

Fuente: Farias (2013, p.280)

A continuación, se presentan ejemplos de paráfrasis definidoras elaboradas para el portugués de acuerdo con este modelo, destacándose que no existe la obligatoriedad de llenarse todos los slots generados en el esquema de definición:

ira sentimento de exaltação intenso e violento, provocado por [uma contrariedade], que leva à privação do uso da razão medo sentimento de apreensão provocado por [algo] (potencialmente) perigoso/prejudicial (FARIAS, 2013, p. 280 , subrayado original).

\footnotetext{
${ }^{24}$ Se han considerado los datos de la FrameNet del inglés, porque aún no se han generado los frames emoções y SENTIMENTO para la FrameNet del portugués. Sin embargo, se argumenta que ello no influye de manera decisiva en los resultados obtenidos. En primer lugar, los frames, en su gran mayoría, se traducen directamente de la FrameNet del inglés. En segundo lugar, las divergencias entre las FrameNets de distintas lenguas, cuando las hay, son producto de las diferencias tipológicas y genéticas entre la lengua de partida (el inglés) y la lengua de llegada (la lengua de la FrameNet que se está construyendo), y raras veces se deben a hechos culturales, así que la estructura de los frames, en general, no se ve afectada en su esencia.

${ }^{25}$ Traducido y adaptado del portugués.
} 
A modo de conclusión, se advierte que:

a) la metodología propuesta puede aplicarse con relativo éxito a la elaboración de esquemas de definición de un gran número de unidades léxicas; sin embargo, hay que reconocer que este modelo no posee valor universal. Por un lado, están las unidades léxicas que se dejan definir sin grandes dificultades mediante el modelo de genus proximum et differentia(e) specifica(e) (como lo demuestra, por ejemplo, el contraste entre los lexemas hombre, mujer, niño, niña). Por otro, están también las unidades léxicas cuyas paráfrasis incluyen elementos de naturaleza extralingüística, con base en un modelo prototípico de definición, sin la necesidad de recurrir a los frames (por ejemplo, las definiciones ostensivas de colores, animales, plantas, etc.);

b) la metodología propuesta representa un intento de formular criterios tan sistemáticos - y por tanto objetivos - como posible para la selección de las informaciones que deben entrar a componer las paráfrasis definidoras (con especial atención a los sustantivos que designan sentimientos/emociones). Sin embargo, hay que reconocer que erradicar la subjetividad en el ámbito de la elaboración de las paráfrasis definidoras sigue siendo un desiderátum. Ello se debe, al menos en parte, a que no existe un modelo de descripción del significado aplicable directamente a la lexicografía (HARRAS, 1991, p. 30; HERBST; KLOTZ, 2003, p. 33). En lo que concierne específicamente a la FrameNet, aunque está orientada lexicográficamente (STEINER, 2004), parece haberse concebido como un instrumento lexicográfico per se, que se puede utilizar en conjunto o, incluso, como alternativa a las obras lexicográficas "tradicionales", y no como un posible material de apoyo lexicográfico (elaboración de obras lexicográficas) y/o metalexicográfico (elaboración de una teoría del diccionario). Por fin, hace falta señalar que la FrameNet es una herramienta en construcción -especialmente en lo que atañe a su adaptación a otras lenguas que no sean el inglés-; su aplicación (meta)lexicográfica seguramente conllevaría una serie de dificultades hasta cierto punto impredecibles. 


\section{Consideraciones finales}

Este estudio posee un carácter exploratorio y presenta el estadio inicial de las investigaciones acerca de la definición de sustantivos que designan sentimientos/emociones.

Hasta ahora lo único que se ha podido sacar en claro mediante el análisis de las definiciones de sentimientos/emociones en diccionarios de lengua es que: (a) el modelo tradicional no presenta una solución satisfactoria para la definición de esta clase de palabras; y (b) la incorporación de informaciones extralingüísticas en las paráfrasis definidoras -aunque necesaria- exige una sistematización. En relación con el segundo problema, nos preocupamos justamente por establecer una metodología que nos permita identificar los rasgos extralingüísticos pertinentes en la definición de sentimientos/emociones y, al mismo tiempo, proponer un patrón para su presentación en las paráfrasis. Tanto el metalenguaje semántico natural como la semántica de marcos ofrecen importantes contribuciones al establecimiento de esta metodología; sin embargo, cada uno de los modelos también presenta limitaciones que no permiten obtener resultados completamente exitosos.

\section{Referencias}

ALBRECHT, J. Fünf Thesen zur Kognitiven Semantik. In: HOINKES, U.; DIETRICH, W. (Hrsg.). Kaleidoskop der lexikalischen Semantik. Tübingen: Narr, 1997. p. 19-39.

. El paradigma incompleto de E. Coseriu: Tarea pendiente para la tercera generación. Odisea, Almería, v. 3, p. 41-54, 2003.

ALGEMEEN NEDERLANDS WOORDENBOEK. Disponible en: $<$ http://anw.inl.nl/search $>$. Acceso en el: 18 jun. 2015.

ALVAR EZQUERRA, M.; GILI GAYA, S. Vox diccionario general ilustrado de la lengua española. Barcelona: Bibliograf, 1997.

ATKINS, B. T. S.; RUNDELL, M. The Oxford guide to practical lexicography. Oxford: OUP, 2008.

BALDINGER, K. Teoría semántica. Hacia una semántica moderna. 2. ed. Madrid: Ediciones Alcalá, 1977.

BALLMER, T.; BRENNENSTUHL, W. An empirical approach to frame theory: verb thesaurus organization. In: EIKMEYER, H.-J.; RIESER, H. 
(Eds.). Words, worlds, and contexts: new approachs in word semantics. Berlin; New York: Walter de Gruyter, 1981. p. 297-319.

BOAS, H. C. Frame Semantics as a framework for describing polysemy and syntactic structures of English and German motion verbs in contrastive computational lexicography. In: RAYSON, P. et al. (Eds.). Proceedings of corpus linguistics. Lancaster: LU Press, 2001. p. 64-73.

. Bilingual FrameNet dictionaries for machine translation. In: INTERNATIONAL CONFERENCE ON LANGUAGE RESOURCES AND EVAlUATION, 3, 2002, Las Palmas. Proceedings... Las Palmas: Universidad de Las Palmas de Gran Canaria, 2002. p. 1364-1371. Disponible en: <http://www.icsi.bekerley.edu/pubs/ai/ bilingualframenet02.pdf>. Acceso el: 9 nov. 2012.

. Wie viel Wissen steckt in Wörterbüchern? Eine framesemantische Perspektive. Zeitschrift für angewandte Linguistik, Mannheim, n. 58, p. 75-97, 2013. Disponible en: <http://pub.idsmannheim.de/extern/zfal/zfal2013-1.html >. Acceso el: 18 jun. 2015.

BOSSE. T.; JONKER, C. M.; TREUR, J. Formalisation of Damasio's theory of emotion, feeling, and core consciousness. Consciousness and Cognition, Amsterdam, v. 17, n. 1, p. 94-113, 2008. Disponible en: $<$ http://www.few.vu.nl/ T.Bosse/papers/CC-damasio.pdf $>$. Acceso el: 10 ago. 2015.

BUGUEÑO MIRANDA, F. Para uma taxonomia de paráfrases explanatórias. Alfa, São Paulo, v. 53, n. 1, p. 243-260, 2009.

BUGUEÑO MIRANDA, F.; FARIAS, V. S. Panorama crítico dos dicionários escolares brasileiros. Lusorama, Frankfurt am Main, v. 77/78, p. 29-78, 2009.

. Princípios para o desenvolvimento de uma teoria da definição lexicográfica. Alfa, São Paulo, v. 55, n. 1, p. 31-61, 2011a. Disponible en: $<$ http://seer.fclar.unesp.br/alfa/article/view/4167/3765>. Acceso el: 10 jun. 2015.

. Sobre las palabras y su clasificación según su contenido. Los problemas para el lexicógrafo. RFULL, La Laguna, n. 29, p. 9-19, 2011b. Disponible en: <http://publica.webs.ull.es/upload/REV\%20 FILOLOGIA/29-2011/01\%20Bugue $\%$ C3\%B10\%20Miranda\%20y\%20 otra.pdf $>$. Acceso el: 7 jun. 2015. 
. Da microestrutura em dicionários semasiológicos do português e seus problemas. Estudos da Lingua (gem), Vitória da Conquista, v. 9, p. 36-69, 2011c. Disponible en:

$<$ http://cpelin.org/estudosdalinguagem/ojs/index.php/ estudosdalinguagem/article/viewFile/121/281>. Acceso el: 10 jun. 2015.

. Los subsidios de tres teorías semánticas para la generación de definiciones lexicográficas. RFULL, La Laguna, n. 31, p. 19-34, 2013a. Disponible en: <http://publica.webs.ull.es/upload/REV\%20 FILOLOGIA/31-2013/Filolog\%C3\%ADa\%2031-3013.pdf $>$. Acceso el: 10 jun. 2015.

. Teorías semánticas y definición lexicográfica. Análisis de las paráfrasis explicativas de los diccionarios generales de lengua española. Cadernos de Tradução, Florianópolis, n. 32, v. 2, p. 183225, 2013b. Disponible en: <https://periodicos.ufsc.br/index.php/ traducao/article/view/2175-7968.2013v2n32p183/25500>. Acceso el: 1 jun. 2015.

CASARES, J. Introducción a la lexicografía moderna. 3. ed. Madrid: Consejo Superior de Investigaciones Científicas, 1992.

COSERIU, E. Tesis acerca del "significado". Lexis, Lima, v. 22, n. 1, p. 83-86, 1998.

CUENCA, M. J.; HILFERTY, J. Introducción a la lingüistica cognitiva. Barcelona: Ariel, 2011.

EKMAN, P. Are the basic emotions? Psychological Review, Washington, v. 99, n. 3, p. 550-553, 1992.

FARIAS, V. S. Fundamentos para uma teoria geral dos mecanismos explanatórios: Objetivos, metodologia e primeiros resultados da pesquisa. Cadernos do IL (UFRGS), Porto Alegre, v. 40, p. 45-65, 2010. Disponible en: $<$ http://seer.ufrgs.br/cadernosdoil/article/view/24900>. Acceso el: 15 ago. 2015.

. Aplicação da semântica das condições de verdade à redação das definições nos dicionários semasiológicos. RBLA, Belo Horizonte, v. 12, n. 1, p. 181-204, 2012. Disponible en: <http://www.scielo.br/pdf/rbla/ v12n1/a10v12n1>. Acceso el: 17 jun. 2015.

. Sobre a definição lexicográfica e seus problemas. Fundamentos para uma teoria geral dos mecanismos explanatórios em dicionários 
semasiológicos. 2013. 399 f. Tese (Doutorado em Lexicografia e Terminologia) - Instituto de Letras, Universidade Federal do Rio Grande do Sul, Porto Alegre, 2013. Disponible en: <https://www.lume.ufrgs.br/ bitstream/handle/10183/90167/000913147.pdf? sequence $=1>$. Acceso el: 17 jun. 2015.

FERREIRA, A. B. et al. Novo dicionário Aurélio da língua portuguesa. 4. ed. Curitiba: Positivo, 2009. 1 CD-ROM.

FILLMORE, C. J. Frame semantics. In: THE LINGUISTIC SOCIETY OF KOREA (Ed.). Linguistics in the morning calm. Seoul: Hanshin, 1982. p. 111-137.

FILLMORE, C. J.; ATKINS, B. T. S. Starting where the dictionaries stop: The challenge of Corpus Lexicography. In: ATKINS, B. T. S.; ZAMPOLLI, A. (Eds.). Computational Approaches to the Lexicon. Oxford; New York: OUP, 1994. p. 349-393.

FILLMORE, C. J. et al. Background to FrameNet. International Journal of Lexicography, Oxford, v. 16, n. 3, p. 235-250, 2003.

FONTAINE, J. R. J. et al. The world of emotions is not two-dimensional. Psychological Science, v. 18, n. 12, p. 1050-1057, 2007.

FONTENELLE, T. WordNet, FrameNet and other semantic networks in the International Journal of Lexicography - The net result? International Journal of Lexicography, Oxford, v. 25, n. 4, p. 437-449, 2012.

FRIEDENBERG, J.; SILVERMAN, G. Cognitive science. An introduction to the study of mind. 2. ed. Los Angeles: SAGE, 2012.

GAO, J. Basic cognitive experiences and definitions in the Longman Dictionary of Contemporary English. International Journal of Lexicography, Oxford, v. 26, n. 1, p. 58-89, 2013.

GARCÍA MARQUEZ, G. La vaina de los diccionarios. El País, Madrid, 19 mayo 1982. Disponible en: <http://elpais.com/diario/1982/05/19/ opinion/390607204_850215.html>. Acceso el: 15 ago. 2015.

GEERAERTS, D. The lexicographical treatment of prototypical polysemy. In: TSOHATZIDIS, S. L. (Ed.). Meanings and prototypes. Studies in linguistic categorization. London; New York: Routledge, 1990. p. 195-210.

. The definitional practice of dictionaries and the Cognitive Semantic conception of polysemy. Lexicographica, Oxford, v. 17, p. 6-21, 2001. 
. Meaning and definition. In: STERKENBURG, P. (Ed.). $A$ practical guide to Lexicography. Amsterdam; Philadelphia: John Benjamins, 2003. p. 83-93.

. Lexicography. In: GEERAERTS, D.; CUYCKENS, H. (Eds.). The Oxford Handbook of Cognitive Linguistics. Oxford; New York: OUP, 2007. p. 1160-1174.

. Theories of lexical semantics. New York: OUP, 2010.

GODDARD, C. Semantic analysis. A practical introduction. 2. ed. Oxford: OUP, 2010.

HAENSCH, G. et al. La lexicografía. Madrid: Gredos, 1982.

HANKS, P. Definitions and Explanations. In: HARTMANN, R. R. K. (Ed.). Lexicography. Critical concepts. London; New York: Routledge, 2003. p. 191-206.

HARRAS, G. Zugänge zu Wortbedeutung. In: HARRAS, G. et al. (Hrsgn.). Wortbedeutung und ihre Darstellung im Wörterbuch. Band 3. Berlin; New York: Walter de Gruyter, 1991. p. 3-96.

HERBST, T.; KLOTZ, M. Lexikografie. Paderborn: Ferdinand Schöningh, 2003.

HORNBY, A. S. Oxford Advanced Learner's Dictionary. 7. ed. Oxford: OUP, 2005.

HOUAISS, A. et al. Dicionário Houaiss da língua portuguesa. Rio de Janeiro: Objetiva, 2009. 1 CD-ROM.

INSTITUT FÜR DEUTSCHE SPRACHE. Online WortschatzInformationssystem Deutsch. Disponible en: $<\mathrm{http}: / /$ www.owid.de/ elexiko_/index.html>. Acceso el: 18 jun. 2013.

ISTITUTO DELLA ENCICLOPEDIA ITALIANA. Vocabolario Treccani della lingua italiana. Roma: Istituto della Enciclopedia Italiana, 1998. Disponible en: <www.treccani.it/vocabolario/>. Acceso el: 18 jun. 2015.

JÄGER, L.; PLUM, S. Probleme der Beschreibung von Gefühlswörtern im allgemeinen einsprachigen Wörterbuch. In: HAUSMANN, F. J. et al. (Hrsgn.). Wörterbücher, Dictionaries, Dictionnaires. Ein internationales Handbuch zur Lexikographie. Band 1. Berlin; New York: Walter de Gruyter, 1989. p. 849-855. 
KAILUWEIT, R. Grammatica e semantica dei verbi di sentimento. Un'analisi basata sulla Role and Reference Grammar. In: BURR, E. (Ed.). Tradizione e Innovazione. Dall'italiano, lingua storica e funzionale, alle altre lingue. Firenze: Franco Cesati, 2009. p. 265-280.

KAMMERER, M. Zur framebasierten lexikographischen Bedeutungsbeschreibung von substantivischen Lemmazeichen. Lexicographica, Oxford, v. 15, p. 229-263, 1999.

KILGARRIFF, A. I don't believe in word senses. Computers and the Humanities, Oxford, v. 31, p. 91-113, 1997.

. Word senses. In: AGIRRE, E.; EDMONDS, P. (Eds.). Word sense disambiguation: algorithms and applications. Dordrecht, T.N.: Springer, 2007. p. 29-46.

KLEIBER, G. La sémantique du prototype. Catégories et sens lexical. 2. ed. Paris: PUF, 2004.

KLOSA, A. et al. Benutzerwünsche und Meinungen zu einer optimierten Wörterbuchpräsentation - Ergebnisse einer Onlinebefragung zu elexiko. Mannheim: Institut für deutsche Sprache/Online publizierte Arbeiten zur Linguistik, 2011. Disponible en: < http://pub.ids-mannheim.de/laufend/ opal/pdf/opal2011-3.pdf $>$. Acceso el: 1 nov. 2013.

KOEVA, S. Lexicon and Grammar in Bulgarian FrameNet. In: INTERNATIONAL CONFERENCE ON LANGUAGE RESOURCES AND EVALUATION, 7, 2010, Valletta. Proceedings... Valletta: European Language Resources Association, 2012. p. 324-330 Disponible en: <http://hnk.ffzg.hr/bibl//rec2010/pdf/705_Paper.pdf > Acceso el: 24 jun. 2013.

KÖVECSES, Z. The conceptual structure of happiness. In: TISSARI, H. et al. (Eds.). Hapinnes: cognition, experience, language. Studies across Disciplines in the Humanities and Social Sciences. Helsinki: Helsinki Collegium for Advanced Studies, 2008. p. 131-143. Disponible en: $<$ http://www.helsinki.fi/collegium/e-series/volumes/volume_3/09 Kovecses 2008 3.pdf $>$. Acceso el: 6 nov. 2013.

LAKOFF, G. Women, fire, and dangerous things. What categories reveal about the mind. Chicago; London: The University of Chicago, 1990.

LARA, L. F. Teoría del diccionario monolingüe. México, D.F.: El Colegio de México, 1996. 
LINDQUIST, K. A. et al. Language and the perception of emotion. Emotion, Boston, v. 6, n. 1, p. 125-138, 2006.

LITTRÉ, E. Dictionnaire de la langue française (en ligne). 1863-1877. Disponible en: <www.littre.reverso.net/dictionnaire-français/>. Acceso el: 18 jun. 2015.

MARTÍNEZ DE SOUZA, J. Diccionario de lexicografía práctica. Barcelona: Bibliograf, 1995.

MÉVEL, J.-P.; GAILLARD, B. (Dir.). Dictionnaire Hachette. Paris: Hachette, 2012.

MICHAELIS. Moderno dicionário da língua portuguesa. São Paulo: Melhoramentos, 1998.

MOERDIJK, F. Frames and semagrams. Meaning description in the general Dutch dictionary. In: EURALEX INTERNATIONAL CONGRESS, 13, 2008, Barcelona. Proceedings... Barcelona: Universitat Pompeu Fabra/Documenta Universitaria, 2008. p. 561-569. Disponible en: <http://www.euralex.org/elx_proceedings/Euralex2008/042 Euralex 2008 Fons\%20Moerdijk Frames\%20and\%20Semagrams Meaning $\% 20 \bar{D}$ escription $\% 20$ in $\% \overline{2} 0$ the $\% 20$ General\%20Dutch $\% 2 \overline{0}$ Dictionary.pdf $>$. Acceso el: 27 mayo 2013.

MOERDIJK, F. et al. Accessing the ANW dictionary. In: WORKSHOP ON COGNITIVE ASPECTS ON THE LEXICON (COGALEX), 1, 2008, Manchester. Proceedings... Manchester: Association for Computational Linguistics, 2008. p. 18-24. Disponible en: $<$ http:// www.aclweb.org/anthology/W/W08/W08-19.pdf $>$. Acceso el: 1 nov. 2013.

MOERDIJK, F. et al. Algemeen Nederlands Woordenboek. Disponible en: $<$ http://anw.inl.nl/search>. Acceso el: 18 jun. 2015.

MOLINER, M. Diccionario de uso del español. Madrid: Gredos, 2001. 1 CD-ROM.

MÜLLER-SPITZER, C.; MÖHRS, C. First ideas of users-adapted views of lexicographic data exemplified on OWID and elexiko. In: WORKSHOP ON COGNITIVE ASPECTS ON THE LEXICON (COGALEX), 1, 2008, Manchester. Proceedings... Manchester: Association for Computational Linguistics, 2008. p. 39-46. Disponible en: < http://www.aclweb.org/anthology/W/W08/W08-19.pdf>. Acceso el: 1 nov. 2013. 
OGDEN, C. K.; RICHARDS, I. A. The meaning of meaning. 8. ed. New York; London: Routledge, 1956.

OHARA, K. H. Semantic annotations in Japanese FrameNet: Comparing Frames in Japanese and Englisch. In: INTERNATIONAL CONFERENCE ON LANGUAGE RESOURCES AND EVALUATION, 8, 2012, Istambul. Proceedings... Istambul: European Language Resources Association, 2012. p. 1559-1562. Disponible en: $<$ http://www.lrec-conf.org/proceedings/lrec2012/pdf/1053_Paper.pdf $>$. Acceso el: 24 jun. 2013.

OLIVEIRA, A. F. S.; BUGUEÑO MIRANDA, F. V.; SIQUEIRA, M. $\mathrm{O}$ tratamento da polissemia e da homonímia nos learner's dictionaries: subsídios da semântica cognitiva para a disposição das acepções. Alfa, São Paulo, v. 57, n. 1, p. 163-197, 2013. Disponible en: < http://seer.fclar. unesp.br/alfa/article/view/4638/4545>. Acceso el: 10 ago. 2015.

OSTERMANN, C. Cognitive lexicography of emotions terms. In: FJELD, R.V.; TORJUSEN, J.M. (Eds.). EURALEX INTERNATIONAL CONGRESS, 15, 2012, Oslo. Proceedings... Oslo: University of Oslo, 2012. p. 493-501 Disponible en: $<$ http://www.euralex.org/proceedingstoc/euralex_2012>. Acceso el: 24 mayo 2013.

PATOTA, G. Il Garzantino della Lingua Italiana. Lavis: Garzanti Linguistica, 2012.

PLUM, S. Gefühlswörter im Wörterbuch. Überlegungen zur lexikographischen Bedeutungserläuterung des emotionalen Wortschatzes. In: MEDER, G.; DÖRNER, A. (Hrsgn.). Worte, Wörter, Wörterbücher. Lexikographische Beiträge zum Essener Linguistischen Kolloquium. Tübingen: Max Niemeyer, 1992. p. 169-182.

PORTO DAPENA, J. A. Manual de técnica lexicográfica. Madrid: Arco, 2002.

POTTIER, B. A definição semântica nos dicionários. Tradução de Maria Angela Botelho Pereira. In: LOBATO, L. M. P. (Ed.). A semântica na linguística moderna: o léxico. Rio de Janeiro: Francisco Alves, 1977. p. 21-31. PRIETO, S. Inclusión de los papeles semánticos de FrameNet en DiCE. In: EURALEX INTERNATIONAL CONGRESS, 13, 2008, Barcelona. Proceedings... Barcelona: Universitat Pompeu Fabra/ Documenta Universitaria, 2008. p. 1393-1399. Disponible en: <http:// www.euralex.org/elx_proceedings/Euralex2008/144_Euralex_2008 
Sabela\%20Prieto\%20Gonzalez_Inclusion $\% 20$ de $\% 201$ os\%20papeles $\% 20$ semanticos\%20de\%20FrameNet\%20en\%20DiCE.pdf $>$. Acceso el: 24 mayo 2013.

PUTNAM, H. La sémantique est-elle possible? In: CHAURAND, J.; MAZIÈRE, F. (Eds.). La définition. Paris: Larousse, 1990. p. 292-304.

RAE. Diccionario de la lengua española. 22. ed. Madrid: Espasa-Calpe, 2001. Disponible en: <www.rae.es>. Acceso el: 18 jun. 2015.

REY, A. Le lexique: images et modèles du dictionnaire à la lexicologie. Paris: Armand Colin, 1977.

REY-DEBOVE, J. La définition lexicographique: recherches sur l'équation sémique. Cahiers de Lexicologie, Cergy-Pontoise, v. 8, n. 1, p. 71-94, 1966.

. Étude linguistique et sémiotique des dictionnaires français contemporains. Paris: Mouton, 1971.

ROBERT, P. Le Nouveau Petit Robert: Dictionnaire alphabétique et analogique de la langue française. Paris: Le Robert, 2001.

ROSCH, E. Principles of categorization. In: ROSCH, E.; LLOYD, B. B. (Eds.). Cognition and categorization. Hillsdale, N.J.: Lawrence Erlbaum, 1978. p. 27-48.

RUNDELL, M. More than one way to skin a cat: Why full-sentence definitions have not been universally adopted. In: FONTENELLE, T. (Ed.). Practical lexicography: a reader. Oxford: OUP, 2008. p. 197-209.

SALOMÃO, M. M. M. FrameNet Brasil: um trabalho em progresso. Calidoscópio, São Leopoldo, v. 7, n. 3, p. 171-182, 2009. Disponible en: $<$ http://revistas.unisinos.br/index.php/calidoscopio/article/view/4870>. Acceso el: 3 nov. 2013.

SECO, M. Estudios de lexicografía española. 2. ed. Madrid: Gredos, 2003.

SECO, M. et al. Diccionario del español actual. 2. ed. Madrid: Santillana, 2011.

SINCLAIR, J. (Ed.). Collins COBUILD advanced learner's dictionary. Glasgow: HarperCollins, 2003. 1 CD-ROM.

STEINER, P. FrameNet und WordNet. Perspektiven für die Verknüpfung zweier lexikalisch-semantischer Netze. LDV-Forum, Trier, v. 19, n. 
1/2, p. 143-154, 2004. Disponible en: <http://www.jlcl.org/2004_ Doppelheft/143-154_Steiner.pdf $>$. Acceso el: 9 nov. 2012.

ULLMANN, S. Semantics. An introduction to the science of meaning. Oxford: Basil Blackwell, 1964.

WEGNER, I. Lexikographische Definition und Frame-Theorie im allgemeinen einsprachigen Wörterbuch. In: HAUSMANN, F. J. et al. (Hrsgn.). Wörterbücher, dictionaries, dictionnaires. Ein internationales Handbuch zur Lexikographie. Band I. Berlin; New York: Walter de Gruyter, 1989. p. 893-899.

WIEGAND, H. E. Die lexikographische Definition im allgemeinen einsprachigen Wörterbuch. In: HAUSMANN, F. J. et al. (Hrsgn.). Wörterbücher, Dictionaries, Dictionnaires. Ein internationales Handbuch zur Lexikographie. Band 1. Berlin; New York: Walter de Gruyter, 1989. p. 530-573.

WIERZBICKA, A. Lexicography and conceptual analysis. Ann Arbor, MI: Karoma, 1985.

. Semantics, culture, and cognition: Universal human concepts in culture-specific configurations. New York; Oxford: OUP, 1992a.

. Defining emotion concepts. Cognitive Science, Austin, v. 16, p. 539-581, $1992 \mathrm{~b}$.

. Emotions across languages and cultures. Cambridge: CUP, 1999. WOTJAK, G. Bedeutung und Kognition. Überlegungen im Spannungsfeld zwischen lexikalischer und kognitiver Semantik. In: HOINKES, U.; DIETRICH, W. (Hrsgn.). Kaleidoskop der lexikalischen Semantik. Tübingen: Narr, 1997. p. 31-59.

ZINGARELLI, N. Vocabolario della lingua italiana. Bologna: Zanichelli, 2011. 